“@ 2018 IEEE. Personal use of this material is permitted. Permission from IEEE must be obtained for all other uses, in any current or future media, including reprinting/republishing this material for advertising or promotional purposes, creating new collective works, for resale or redistribution to servers or lists, or reuse of any copyrighted component of this work in other works." 


\title{
A Novel Low-Profile Wideband Reconfigurable CP Antenna Array
}

\author{
Shu-Lin Chen ${ }^{1}$, Wei Lin ${ }^{1}$, Pei-Yuan Qin ${ }^{1}$, Y. Jay Guo ${ }^{1}$, and Richard W. Ziolkowski ${ }^{1,2}$ \\ ': Global Big Data Technologies Centre, University of Technology Sydney, Sydney, Australia \\ Email: ShulinChen.UTS@gmail.com* \\ ${ }^{2}$ : Department of Electrical and Computer Engineering, University of Arizona, Tucson, USA
}

\begin{abstract}
For future wireless communications, costeffective, low-profile circular polarization (CP) antennas with wide bandwidth and high directivity are highly desirable to increase system capacity and suppress polarization mismatch. In this paper, a wideband circular polarization antenna array integrated with a polarization-independent artificial magnetic conductor (AMC) is reported that meets the demands. First, a wideband $\mathrm{CP}$ reconfigurable antenna with a pair of crossbowtie radiators and a metal ground is presented to achieve a fractional bandwidth of $35.9 \%$. By replacing the metal ground with a polarization-independent AMC ground, the antenna profile is reduced from $0.25 \lambda_{0}$ to $0.05 \lambda_{0}$ with only a slight bandwidth decrease. A wideband $\mathrm{CP}$ reconfigurable 4-element linear array is achieved using four of those elements. It is low profile $\left(0.05 \lambda_{0}\right)$, and has a wide operating bandwidth $(21.7 \%)$, and a high realized gain $(13 \mathrm{dBic})$.
\end{abstract}

Index Terms - antenna array, artificial magnetic conductor (AMC), circular polarization, polarization reconfigurable antenna

\section{INTRODUCTION}

The performance demands associated with future wireless communications are evolving towards extremely large data volumes and system capacities. Polarization reconfigurable antennas that can alter the polarization in realtime using switching elements such as PIN diodes have received increasingly growing attention to meet those emerging environment requirements. They offer many advantageous features including expanding data capacity and reducing polarization mismatch [1]-[4]. For instance, circular polarization $(\mathrm{CP})$ reconfigurable antennas with moderate and high gains are highly desirable for satellite applications.

In the last decade, substantial efforts have been made to realizing CP reconfigurability, i.e., switching between lefthanded circular polarization (LHCP) and right-handed circular polarization (RHCP) [5]-[7]. Depending on the mechanisms employed to reconfigure those $\mathrm{CP}$ states, the research reported in the literature can be classified into two main groups. One employs switchable feed network, e.g., polarization switching in [5] is enabled by utilizing a reconfigurable cross-dipole feed integrated with a pair of perturbation lines on a magneto-electric dipole antenna. The other employs reconfigurable radiators, i.e., reconfiguring the length of the U-slot arms of the system reported in [7] enables switchable CP states. It is noted that the inherently narrow bandwidth feature of the feed network and/or the radiator significantly limits the antenna's operating bandwidth. No more than 7\% fractional CP-operation (i.e., overlapped impedance and AR) fractional bandwidth (FBW) has been realized among previous designs. It is well known that an antenna's operating bandwidth has a large effect on the system capacity. Thus, the realization of wideband CP reconfigurable antenna is highly desirable for future wireless communication systems. For instance, in [8] a dipole antenna was positioned above a polarization-rotation artificial magnetic conductor (AMC) structure and integrated with a reconfigurable RF-integrated circuit; it achieved a CPoperation FBW of $15.5 \%$. Another example was reported previously by one of our authors [9]-[10]. A CP-operation FBW of $23.5 \%$ and $20.8 \%$ for the two CP state was achieved using four sequential monopoles with a reconfigurable feed network and a patch antenna with L-shaped feeding probes and reconfigurable feed lines, respectively. However, all of these designs incorporate relatively complex structures and are very difficult to be applied in an array configuration to achieve a high directivity, which is always essential to improve the resolution for satellite systems.

To date, a few wideband $\mathrm{CP}$ reconfigurable antenna arrays have been presented to yield high directivities [11][12]. However, only simulated results are provided in the presented array designs. It should be emphasized that the ideal simulation results are a far cry from the practical measured ones considering the realistic effects of RF switches and DC biasing lines on reconfigurable antenna array prototypes. To the extent of our knowledge, only in a recent work [13], a $1 \times 4$ E-shaped patch antenna array incorporating MEMS switches was fabricated and tested. The CP-operation FBWs were $20 \%$ with a peak gain around 12.8 dBic. Nonetheless, the incorporation of MEMS switches is neither cost-effective nor easy to fabricate.

In this paper, we report the design, fabrication, and measurement of a $1 \times 4 \mathrm{CP}$ reconfigurable antenna array. It achieves a measured CP-operation FBW of $21.7 \%$. It is low profile, $0.05 \lambda_{0}$; has a high directivity of $13 \mathrm{dBic}$; and has low fabrication complexity. To finally accomplish the array, a wideband $\mathrm{CP}$ reconfigurable antenna formed with a pair of cross-bowtie radiators and a metal ground is first introduced. Then, to reduce the antenna profile from $0.25 \lambda_{0}$ to $0.05 \lambda_{0}$, a wideband polarization-independent AMC structure is introduced to replace the metal ground. Finally, a $1 \times 4$ array of these radiators is optimized to realize the desired 
wideband $\mathrm{CP}$-operation reconfigurable array. A prototype was fabricated and measured, confirming the design.

\section{A WIDEBAND CP RECONFIGURABLE ANTENNA WITH METAL GROUND}

The geometry of the proposed wideband CP reconfigurable antenna with metal ground is shown in Fig. 1. Two substrates, Substrate \#1 and Substrate \#2, are employed in this antenna. Both of them are Rogers RT/Duriod 5880 laminate $\left(\varepsilon_{\mathrm{r}}=2.2, \tan \delta=0.0009\right)$ with the thickness of 0.79 $\mathrm{mm}$. The two mirror-symmetric bowtie radiators are printed on the top and bottom of Substrate \#1, respectively. Each radiator consists of a center square pad and two triangular elements connected with a short-arc conductive strip as shown in the top and bottom close-up views in Fig.1. The short-arc conductive strip produces the $90^{\circ}$ phase difference required for $\mathrm{CP}$ radiation. The $\mathrm{CP}$ radiation mechanism associated with this structure originates from the cross-dipole design in [14]. Four diodes are soldered between the center square pads and triangle elements to realize the polarization reconfigurability. A coaxial cable feeds the radiators, i.e., the inner conductor of the coaxial cable connects to the top radiator, and the outer conductor connects to the bottom one. On the top of Substrate \#2, a metal ground plane is placed at a height $0.25 \lambda_{0}$ from Substrate \#1 to act as a reflector and to eliminate the back radiation. On the bottom of Substrate \#2, a printed microstrip line facilitates the realization of a microstrip-to-coax transition. Two DC signals, DC \#1 and $\mathrm{DC} \# 2$, are used to provide the requisite DC biasing voltages.

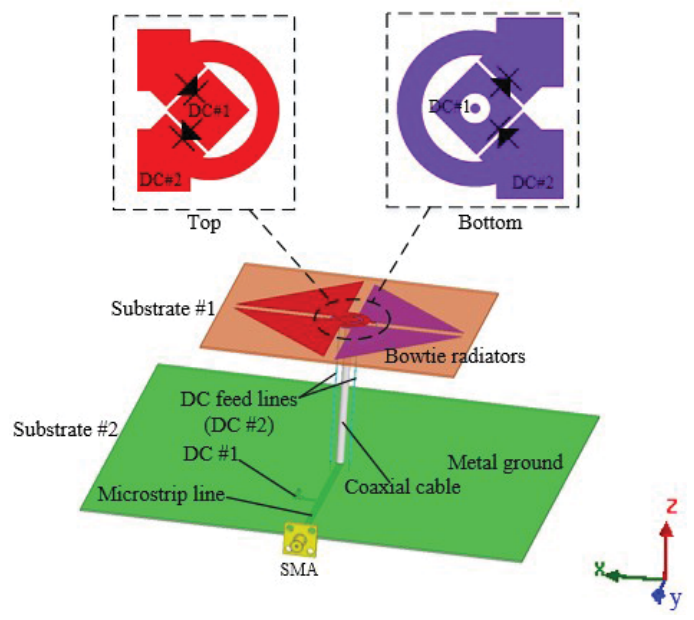

Fig. 1 Geometry of the proposed wideband CP reconfigurable antenna with metal ground.

It should be emphasized that in this design, a very simple switching configuration is employed to achieve the polarization reconfigurability. The layout of the four diodes is shown in a close-up view in Fig. 1. The Bar50-02L PIN diodes from Infineon Technologies with the 0403 surface mount packaging were adopted in this design. Its equivalent circuit model and measured characteristics are reported in the previous works [15], [16]. To illustrate the simplicity of the switch, we consider the LHCP radiation state. When DC $\# 1$ and DC \#2 are energized with $1.5 \mathrm{~V}$ and $0 \mathrm{~V}$ bias voltages, respectively, the two diodes aligned along the $\phi=45^{\circ}$ diagonal are switched on while the other two diodes are off. Thus, the phases of the current on four triangle elements are $0^{\circ},-90^{\circ},-180^{\circ}$, and $-270^{\circ}$, respectively, indicating a clockwise sequential delay. In this case, LHCP radiation is excited. Similarly, one achieves the RHCP state by applying $0 \mathrm{~V}$ and $1.5 \mathrm{~V}$ to $\mathrm{DC} \# 1$ and DC \#2, respectively.

To make the following discussions more clear, this wideband $\mathrm{CP}$ reconfigurable antenna with a metal ground is named Ant \#1. An HFSS simulation model based on Fig. 1 was constructed and used to optimize the design. The obtained final design parameters for Ant \#1 are given in Table I. Due to the symmetry of RHCP and LHCP states, we report the simulated results for only one state.

TABLE I

OPTIMIZED VALUES OF ANT \#1 1 (DIMENSIONS IN MILLIMETERS)

\begin{tabular}{|l|l|l|l|l|l|l|}
\hline Parameter & $L_{\text {rad 1 }}$ & $L_{\text {rad 2 }}$ & $L_{\text {sub1 }}$ & $L_{\text {sub2 }}$ & $D_{\text {d_line }}$ & $W_{\text {d_line }}$ \\
\hline Values $(\mathrm{mm})$ & 37.3 & 60.5 & 100 & 174 & 16 & 2.5 \\
\hline Parameter & $d_{\text {gap }}$ & $H_{\text {anttenna }}$ & $W_{\text {feedline }}$ & $G_{\text {inductor }}$ & $G_{\text {diode }}$ & \\
\hline Values $(\mathrm{mm})$ & 4.4 & 59.6 & 2.6 & 0.5 & 0.4 & \\
\hline
\end{tabular}

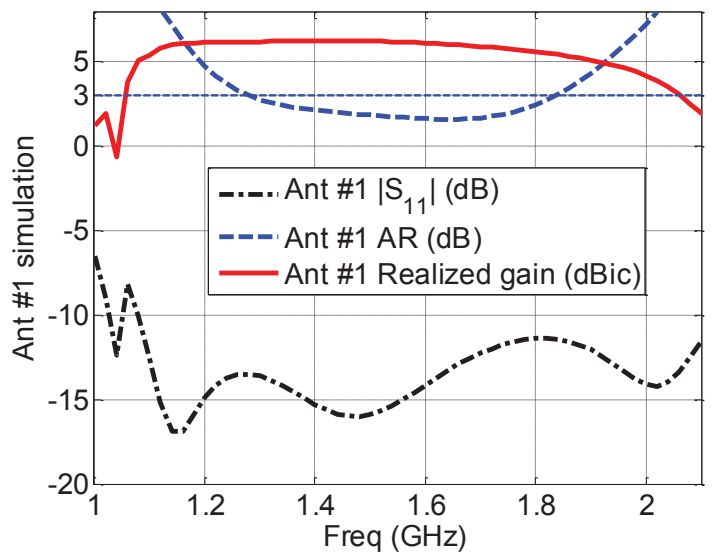

Fig. 2 Simulated $\left|S_{11}\right|$, AR and realized gain values for Ant \#1.

Fig. 2 gives the simulated $\left|S_{11}\right|$, axial ratio (AR), and realized gain values of Ant \#1 versus the frequency. Again note that the $\mathrm{CP}$-operation $\mathrm{FBW}$ is defined by the overlap of the frequency ranges that simultaneously satisfy both $|\mathrm{S} 11| \leq$ -10 and $\mathrm{AR} \leq 3$ and a realized gain variance $\leq 3 \mathrm{dBic}$. It is observed that Ant \#1's CP-operation bandwidth ranges from $1.28 \mathrm{GHz}$ to $1.84 \mathrm{GHz}$, giving a $35.9 \%$ fractional bandwidth. It is worthy to emphasize that this antenna's operating bandwidth is the widest among all the reported designs [5][13], yet has a very simple configuration. Furthermore, the 
antenna yields a flat gain cross the whole operating band with a peak gain of $6.3 \mathrm{dBic}$.

\section{A WIDEBAND CP RECONFIGURABLE ANTENNA WITH AMC GROUND}

It is noted that the incorporation of the metal ground made the antenna's profile (height) to be $0.25 \lambda_{0}$. However, antennas for future wireless systems are desired that can be integrated into a compact and limited space. Thus, a lowprofile characteristic is a prerequisite for antennas applied in most mobile platform communication systems. To reduce the profile of our aforementioned CP antenna, a wideband polarization-independent AMC ground was designed to replace the metal ground. As a result, the antenna's profile was reduced from $0.25 \lambda_{0}$ to $0.05 \lambda_{0}$.

The idea of utilizing an AMC ground plate to achieve a low profile arises from the fact that it will exhibit a $0^{\circ}$ reflection phase. Thus, only a short distance between it and the radiator is required to reflect the back radiation in phase with the source fields.

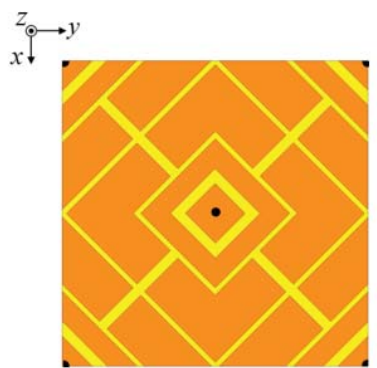

(a)

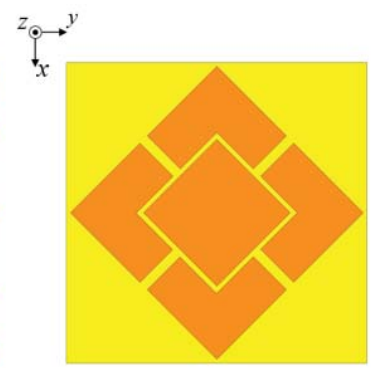

(b)
Fig. 3 Geometry of AMC ground. (a) AMC-A. (b) AMC-B.

The unit cell geometry of our proposed AMC ground is drawn in Fig. 3(a). It is labeled as AMC-A to simplify the following discussion. It is developed from the reported AMC unit cell in [17], which is labeled as AMC-B shown in Fig. 3(b). Based on AMC-B, one uses two steps to achieve AMC-A: 1) Etching a square slot on the center square patch and connecting the patch at its center to the ground plane with a shorting post; 2) Filling four corners of AMC-B with its own quarter pieces.

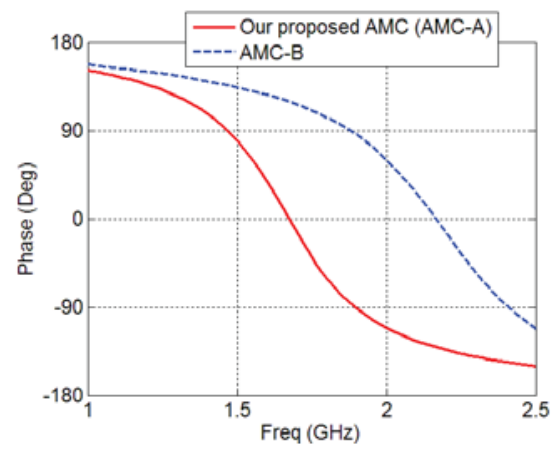

Fig. 4 Reflection phases of AMC-A and AMC-B versus frequency.
Fig. 4 shows the simulated reflection phases of AMC-A and AMC-B versus the excitation frequency for normal incidence. It is noted that the center resonant frequency (zero crossing) of AMC-A is $1.68 \mathrm{GHz}$, red-shifted towards lower frequencies as compared to $2.14 \mathrm{GHz}$ of AMC-B. Thus, for the same working frequency band, AMC-A can achieve a $20 \%$ smaller size with regards to that of AMC-B. The frequency shift can be explained using the parallel LC circuit in [18]. The equivalent effect of filling the four corners is increasing the capacitance in the parallel LC circuit, thus the resonant frequency will be decreased.

Fig. 5 illustrates the design of the wideband CP reconfigurable antenna with $\mathrm{AMC}$ ground obtained by replacing the metal ground in Ant \#1 with a plate consisting of AMC-A cells. Three substrates, Substrate \#1, Substrate $\# 2$, and Substrate \#3, are deployed in this design, printed with the bowtie radiators, AMC ground and microstrip feed network, respectively. Substrate \#1 and Substrate \#3 are Rogers RT/Duriod 5880 laminate $\left(\varepsilon_{\mathrm{r}}=2.2, \tan \delta=0.0009\right)$ with the thickness of $0.79 \mathrm{~mm}$, while Substrate \#2 is produced by Wangling $\operatorname{Ltd}\left(\varepsilon_{\mathrm{r}}=2.2, \tan \delta=0.001\right)$ with the thickness of $8.0 \mathrm{~mm}$. This $\mathrm{CP}$ reconfigurable antenna with AMC ground is named Ant \#2. The switching mechanism for Ant \#2 is the same as described in Ant \#1. The tuned parameters in Ant \#2 are: $L_{\text {rad } 1}=29.8 \mathrm{~mm}, L_{\text {rad } 2}=49.2 \mathrm{~mm}$, $D_{d_{-} \text {line }}=15 \mathrm{~mm}, H=20 \mathrm{~mm}$, while the others are kept the same as in Table I.

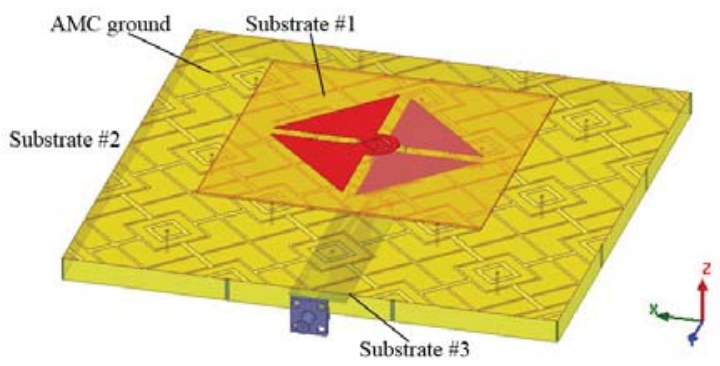

Fig. 5 HFSS simulation model of the wideband CP reconfigurable antenna with AMC ground.

Fig. 6 presents the simulated $\left|S_{11}\right|, A R$, and realized gain values of Ant \#2 versus the frequency. It shows that Ant \#2's CP-operation fractional bandwidth is $26.8 \%$ from 1.42 to $1.86 \mathrm{GHz}$. Compared to Ant \#1, Ant \#2 exhibits a narrower fractional bandwidth, while the profile of Ant \#2 is only $0.05 \lambda_{0}$. This is a tradeoff between the antenna's profile and operating bandwidth. Nonetheless, Ant \#2's operating bandwidth is still wider than any of the other reported designs. The peak realized gain of Ant \#2 is $6.5 \mathrm{dBic}$. 


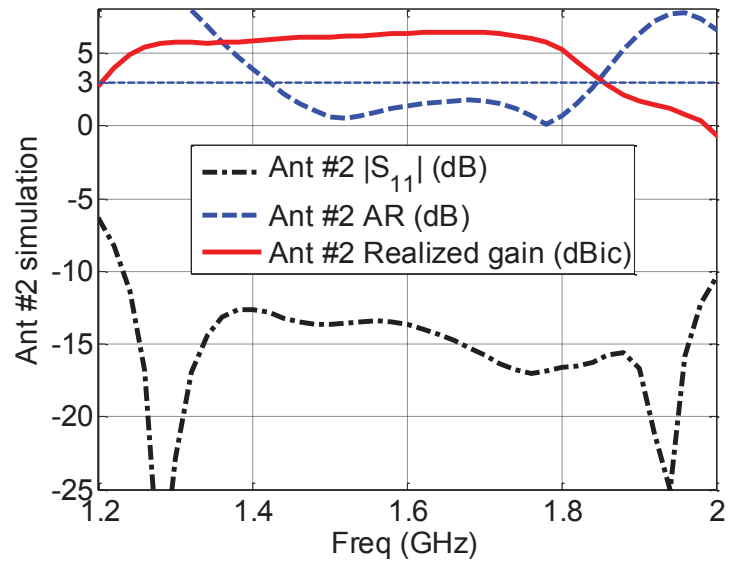

Fig. 6 Simulated performance characteristics of Ant \#2.

\section{WIDEBAND $1 \times 4$ CP RECONFIGURABLE ANTENNA ARRAY WITH AMC GROUND}

Due to the simlicity of Ant \#2, a $1 \times 4$ wideband CP reconfigurable antenna array was designed and simulated by dupilicating four Ant \#2 with a distance $d_{\mathrm{s}}$ separating their centers as shown in Fig. 7. A 1 to 4 Wilkinson power divider was designed to provide equal exicitations for four elements across the target L-band frequency range, 1-2 GHz. This antenna array is named Ant \#3. Again, to switch between its two $\mathrm{CP}$ states, one can just alter the voltages applied on the two DC lines DC \#1 and DC \#2. The optimized parameters for Ant \#3 are: $L_{\mathrm{amc}}=522.0 \mathrm{~mm}, L_{\text {feed }}$ line $=400.0 \mathrm{~mm}, W_{\text {feed line }}=100.0 \mathrm{~mm}, d_{\mathrm{s}}=116.0 \mathrm{~mm}$ while the others are kept the same as Ant \#2.

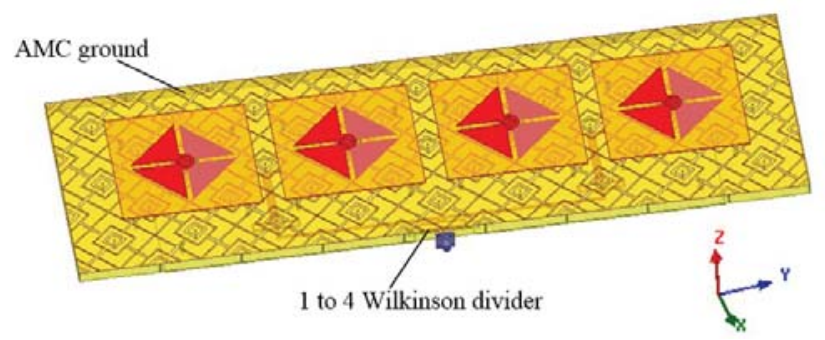

Fig. 7 Simulation model of $1 \times 4$ wideband $C P$ reconfigurable antenna array.

The $1 \times 4$ wideband $\mathrm{CP}$ reconfigurable antenna array (Ant \#3) was fabricated, assembled and measured. Fig. 8 provides the measured $\left|S_{11}\right|$, AR, and realized gain values versus the frequency. Ant \#3's measured CP-operation bandwidth is from 1.44-1.79 GHz, yielding a FBW=21.7\%. For the whole operating band, the gain curve is flat with a maximum gain value of 13.0 dBic. Fig. 9 gives the measured normalized radiation patterns at $1.6 \mathrm{GHz}$. The half-beam widths in the $\mathrm{YZ}$ plane and $\mathrm{XZ}$ plane are $22^{\circ}$ and $73^{\circ}$, respectively. The narrower beam width in the $\mathrm{YZ}$ plane is theoretically reasonable with regards to the one in the $\mathrm{XZ}$ plane due to the large radiating aperture (Four elements) in the $\mathrm{YZ}$ plane (The beamwidth in the $\mathrm{YZ}$ plane is almost four times narrower than the one in the $\mathrm{XZ}$ plane).

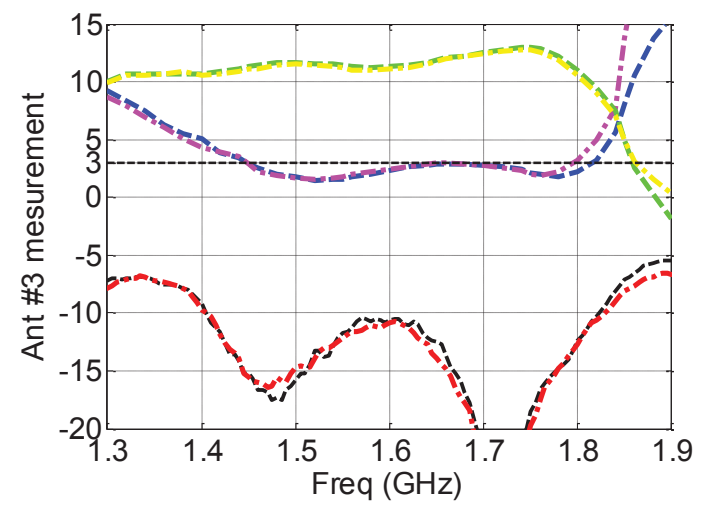

Fig. 8 Measured LHCP and RHCP values of Ant \#3. Legend: $\left|\mathrm{S}_{11}\right|$, Red dash and black dash dot. AR, Blue dash and magenta dash dot. Realized gain, yellow dash and green dash dot.

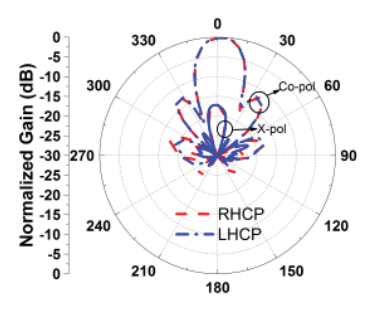

(a)

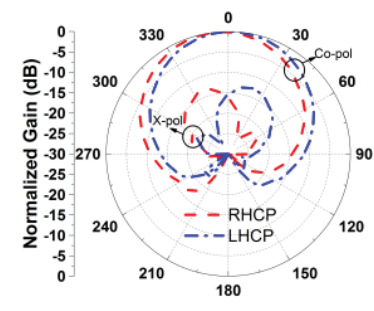

(b)
Fig. 9 Measured normalized radiation patterns at $1.6 \mathrm{GHz}$. (a) YZ plane. (b) XZ plane.

\section{CONCLUSION}

A CP reconfigurable wideband antenna with and without an $\mathrm{AMC}$ ground and a $\mathrm{CP}$ reconfigurable wideband array were presented in this paper. The design of the basic circular polarization reconfigurable antenna with metal ground achieves a $35.9 \% \mathrm{CP}$-operation fractional bandwidth with a simple configuration. The second version with the polarization-independent AMC ground replacement has a much reduced profile from $0.25 \lambda_{0}$ to $0.05 \lambda_{0}$ with the tradeoff that the CP-operation fractional bandwidth also decreases to $26.8 \%$. Then, a $1 \times 4$ array constructed with four of the wideband $\mathrm{CP}$ reconfigurable antenna with $\mathrm{AMC}$ ground elements was fabricated and measured. The low profile was maintained while the gain was increased substantially, to a peak of $13.0 \mathrm{dBic}$. Due to the simple antenna configuration and bias feed network, this array is cost-effective and easyto-fabricate. The realized wideband $\mathrm{CP}$ reconfigurable antennas and arrays of them can be deployed to improve the performance of many wireless systems including $5 \mathrm{G}$ communication and satellite systems. 


\section{REFERENCES}

[1] P.-Y. Qin, Y. J. Guo, Y. Cai, E. Dutkiewicz, and C.-H. Liang, "A reconfigurable antenna with frequency and polarization agility," IEEE Antenna Wireless Propag. Lett., vol. 10, pp. 1373-1376, 2011.

[2] N. Nguyen-Trong, A. Piotrowski, L. Hall, and C. Fumeaux, "A frequency- and polarization-reconfigurable circular cavity antenna," IEEE Antenna Wireless Propag. Lett., vol. 16, pp. 999-1002, 2016.

[3] W. Lin and H. Wong, "Multi-polarization reconfigurable circular patch antenna with L-shaped probes," IEEE Antenna Wirel.Propag. Lett., vol. 16, pp. 1549-1552, 2017.

[4] H. Wong, W. Lin, L. Huitema, and E. Arnaud, "Multi-polarization reconfigurable antenna for wireless biomedical system," IEEE Trans. Biomed. Circuits Syst., vol. 13, no. 3, pp. 652-660, Jun. 2017.

[5] F. Wu and K. M. Luk, "Single-port reconfigurable magneto-electric dipole antenna with quad-polarization diversity," IEEE Trans. Antennas Propag., vol. 65, no. 5, pp. 2289-2296, May 2017.

[6] J.-S. Row, W.-L. Liu, and T.-R. Chen, "Circular polarization and polarization reconfigurable designs for annular slot antennas," IEEE Trans. Antennas Propag., vol. 60, no.12, pp. 5998-6002, Dec. 2012.

[7] P.-Y. Qin, A. R. Weily, Y. J. Guo, and C.-H. Liang, "Polarization reconfigurable U-slot patch antenna," IEEE Trans. Antennas Propag., vol. 58, no. 10, pp. 3383-3388, Oct. 2010.

[8] W. Yang, W. Che, H. Jin, W. Feng, and Q. Xue, "A polarizationreconfigurable dipole antenna using polarization rotation AMC structure," IEEE Trans. Antennas Propag., vol. 63, no. 12, pp. 53055315, Dec. 2015.

[9] W. Lin and H. Wong, "Wideband circular polarization reconfigurable antenna," IEEE Trans. Antennas Propag., vol. 63, no. 12, pp. 59385944, Dec. 2015.

[10] W. Lin and H. Wong, "Wideband circular polarization reconfigurable antenna with L-shaped feeding probes," IEEE Antenna Wireless Propag. Lett., vol. 16, pp. 2114-2117, 2017.

[11] E. Nishiyama, M. Aikawa, and S. Sasaki, "Polarisation switchable slot-ring array antenna," IET Microwaves, Antennas \& Propagation, vol. 2, no.3, pp. 236-241, 2008.

[12] D. V. Navarro-Méndez, L. F. Carrera-Suárez, M. Baquero-Escudero, and H. C. Moy-Li, "Reconfigurable array antenna in LTCC technology," in Proc. 2014 IEEE Antennas and Propagation Society International Symposium (APSURSI2014), Memphis, TN, Jul. 2014, pp. 1670-1671.

[13] J. M. Kovitz, H. Rajagopalan, and Y. Rahmat-Samii, "Design and implementation of broadband MEMS RHCP/LHCP reconfigurable arrays using rotated E-Shaped patch elements," IEEE Trans. Antennas Propag., vol. 63, no. 6, pp. 2497-2507, Jun. 2015.

[14] S. X. Ta, I. Park, and R. W. Ziolkowski, "Crossed dipole antennas: a review.," IEEE Antennas Propagat. Mag., vol. 57, no. 5, pp. 107-122, Oct. 2015.

[15] W. Lin and H. Wong, "Polarization reconfigurable aperture-fed patch antenna and array," IEEE Access, vol. 4, pp. 1510-1517, Apr. 2016.

[16] W. Lin and H. Wong, "Polarization reconfigurable wheel-shaped antenna with conical-beam radiation pattern," IEEE Trans. Antennas Propag., vol. 63, no. 2, pp. 491-499, Feb. 2015.

[17] H. Malekpoor and S. Jam, "Improved radiation performance of low profile printed slot antenna using wideband planar AMC surface," IEEE Trans. Antennas Propag., vol. 64, no. 11, pp. 4626-4638, Nov. 2016.

[18] D. Sievenpiper, L. Zhang, R. F. J. Broas, N. G. Alexopolous, and E. Yablonovitch, "High-impedance electromagnetic surfaces with a forbidden frequency band," IEEE Trans. Microw. Theory Tech., vol. 47, no. 11, pp. 2059-2074, Nov. 1999. 